\title{
Unplanned change from double free flap to a chimeric anterolateral thigh flap in recurrent laryngeal cancer
}

\author{
Sae Hwi Ki ${ }^{1,2}$, \\ Sung Hwan $\mathrm{Ma}^{2}$, \\ Seung Hyun $\mathrm{Sim}^{2}$, \\ Matthew Seung Suk Choi ${ }^{2}$ \\ ${ }^{1}$ Department of Plastic Surgery, Inha \\ University School of Medicine, Incheon; \\ ${ }^{2}$ Department of Plastic Surgery, Inha \\ University Hospital, Incheon, Korea
}

\begin{abstract}
Reconstruction method choice in recurrent head and neck cancer depends on surgical history, radiation therapy dosage, conditions of recipient vessels, and general patient condition. Furthermore, when defects are multiple or three dimensional in nature, reconstruction and flap choice aimed at rebuilding the functional structure of the head and neck are difficult. We experienced successful reconstruction of recurrent laryngeal cancer requiring reconstruction of esophageal and tracheostomy stroma defects using a chimeric two-skin anterolateral thigh flap with a single pedicle.
\end{abstract}

Keywords: Esophagus / Free flaps / Reconstruction / Recurrent

\section{INTRODUCTION}

The choice of reconstruction method after ablative resection of head and neck cancer depends on a variety of factors such as patient age, cancer stage, recipient vessel condition, and flap size and thickness and composition. Furthermore, in patients with recurrent head and neck cancer, particularly in those that have undergone radiotherapy, flap and recipient vessel selection are limited by tumor extent, radiation fibrosis, and surgical scars.

When multiple area reconstruction or three-dimensional (3D) reconstruction is required in recurrent head and neck cancer, flap choice is determined by; general patient condition, degree of cancer metastasis, the surgeon's workhorse flap, number of available recipient vessels, and the character of the flap [1]. When multiple vessels are available, multiple free flaps can be used, but when recipient vessel availability is limited, a local or

\section{Correspondence: Sae Hwi Ki}

Department of Plastic Surgery, Inha University School of Medicine, 27 Inhang-ro,

Jung-gu, Incheon 22332, Korea

E-mail: mdki67@naver.com

Received September 5, 2019 / Revised October 5, 2019 / Accepted October 7, 2019 distant flap (e.g., a pectoralis major myocutaneous or a deltopectoral flap) must be selected. As with the advanced microsurgery technique, and advanced knowledge of flap physiology, surgeon could reconstruct multiple areas or 3D defects using a microsurgical free flap [2-4]. We describe the successful reconstruction of multiple defects in a patient with recurrent laryngeal cancer using a chimeric free flap and discuss possible reconstruction methods in such cases.

\section{CASE REPORT}

A 65-year-old man with a 30-year pack smoking history had undergone left lateral laryngectomy 15 years ago. He had again laryngomicrosurgery for laryngeal and interarytenoid cancers 11 years ago. After 6 years of follow-up, cancer recurred on the right larynx, and chemotherapy and $250 \mathrm{cGy}$ of radiation therapy, and tracheostomy (due to airway stenosis) were performed. The patient had respiratory disturbance 18 months prior to this presentation. Work-up at the time revealed recurrence in the posterior pharynx and neck metastatic lymph nodes, and thus, surgical treatment and postoperative chemotherapy were 
planned. The previous tracheostomy site was severe scarred and the blood supply for need simultaneous reconstruction was poor. A radiologic study showed the external carotid artery appeared normal though narrower than expected.

Total laryngopharyngectomy by ablative surgery and functional neck dissection were performed. After resection, a complete defect of the hypopharynx, upper esophagus, and tracheostomy stroma was observed (Fig. 1). The defect at the tracheotomy stromal site was $8 \times 6 \mathrm{~cm}^{2}$. The initial reconstruction plan involved the use of two flaps, one for the hypopharynx and upper esophagus and the other for the tracheostomy stroma.

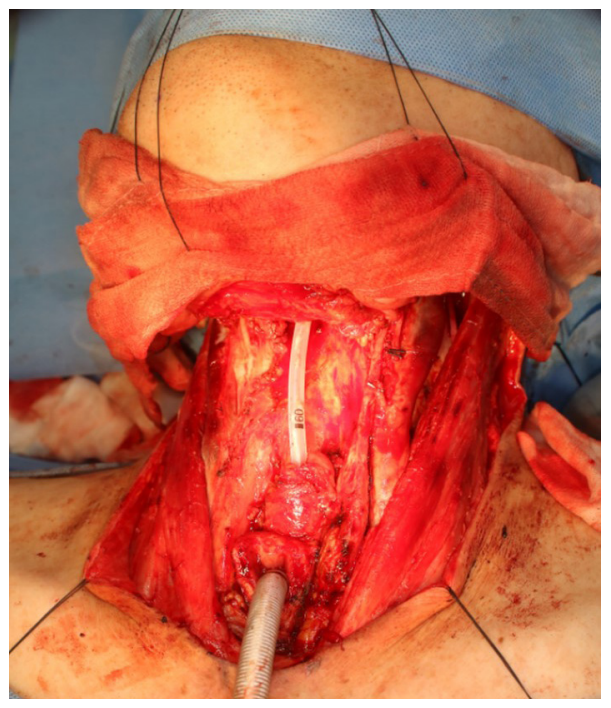

Fig. 1. Circumferential defect of the esophagus and the tracheostomy stromal defect.
Pulsation was present in the external carotid artery, but on resection the facial and lingual arteries and other branches of the external carotid artery (except the superior thyroid artery) were fibrotic with no pulsation or bleeding. The external jugular vein was normal. Because only the superior thyroid artery was available, we should choice the appropriate reconstruction method for multiple area defects. Hand-held Doppler revealed a perforator placed in mid-circle (from anterior superior iliac spine to lateral margin of a patella) on an anterolateral thigh and another perforator on the ipsilateral distal thigh. The size of the anterlateral thigh (ALT) flap was determined to be $12 \times 15 \mathrm{~cm}^{2}$ in the proximal ALT for esophageal reconstruction and an $8 \times 6$ $\mathrm{cm}^{2}$ in the distal ALT for reconstruction of tracheostomy stroma (Fig. 2). We dissected and elevated the ALT flap on the suprafascial plane, the proximal ALT flap was a musculocutaneous perforator flap and the distal ALT flap was a musculocutaneous flap. These ALT flaps were connected with the descending branch of the circumflex femoral artery. The length of pedicle was $6 \mathrm{~cm}$. A vertical suture was placed on the upper ALT flap, which was used to reconstruct the esophagus. The lower ALT flap was used to reconstruct tracheostomy stroma (Fig. 3).

The superior thyroid artery was connected to a descending branch of the lateral femoral circumflex artery by end-to-end anastomosis using nylon 10-0. Two venae comitantes were connected with a branch of the internal jugular vein and no name vein, respectively, on sternocleidomastoid muscle by end-toend anastomosis using nylon 10-0. After the anastomosis, flap blood flow was restored. The donor site was closed by primary closure using a split-thickness skin graft. The ALT flaps sur-
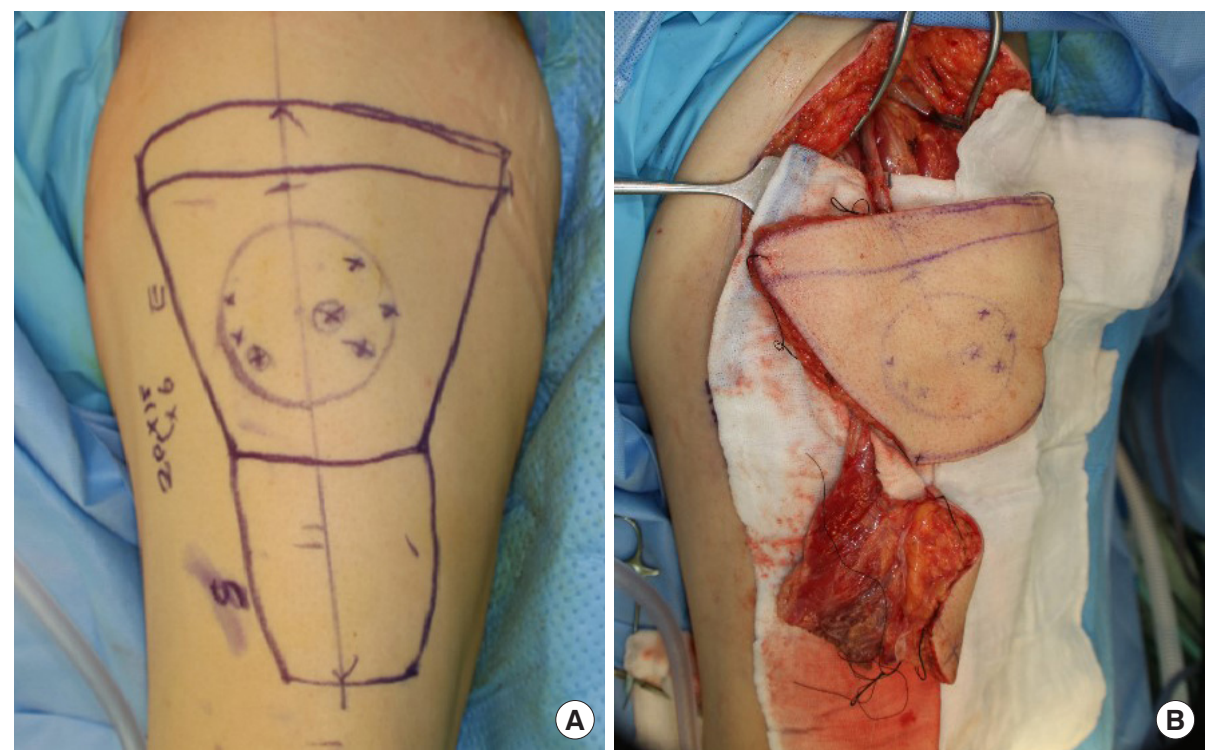

Fig. 2. (A) Design of the two anterolateral flaps with one pedicle. (B) Chimeric flap connected with descending branch of the lateral femoral circumflex artery. 

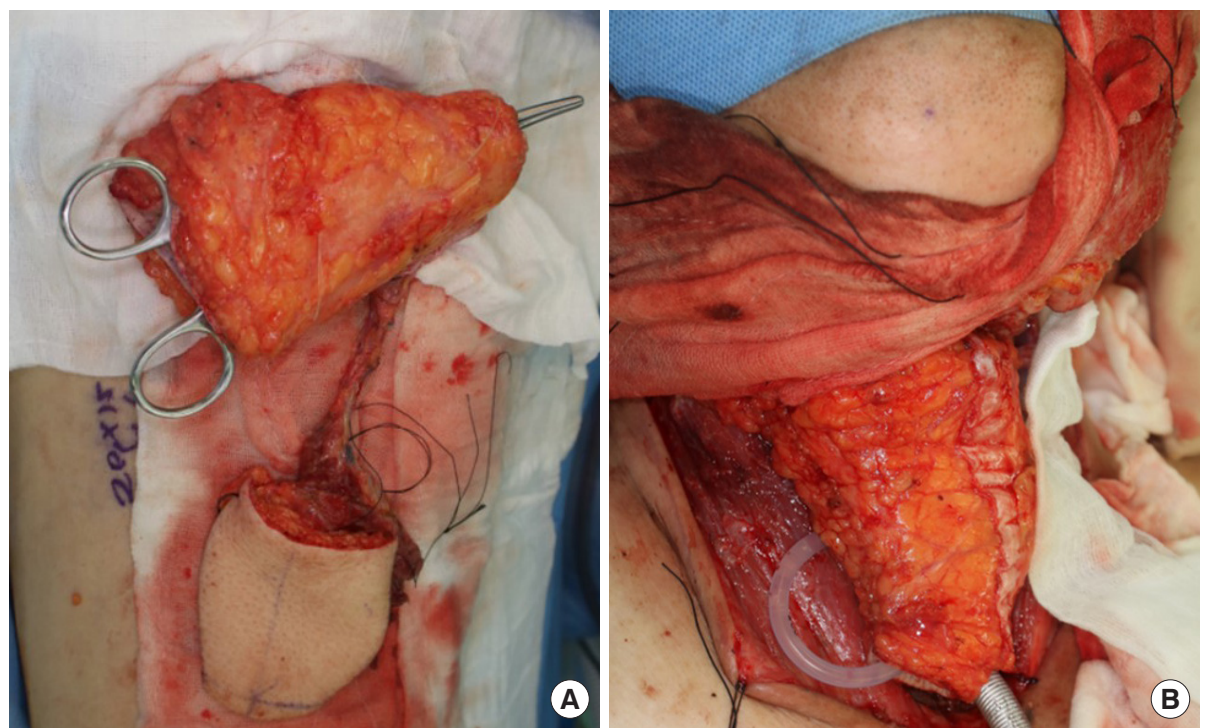

Fig. 3. (A) The upper flap was used for esophagus reconstruction and lower flap for tracheostomy stroma reconstruction. (B) The upper flap was placed in the esophagus.
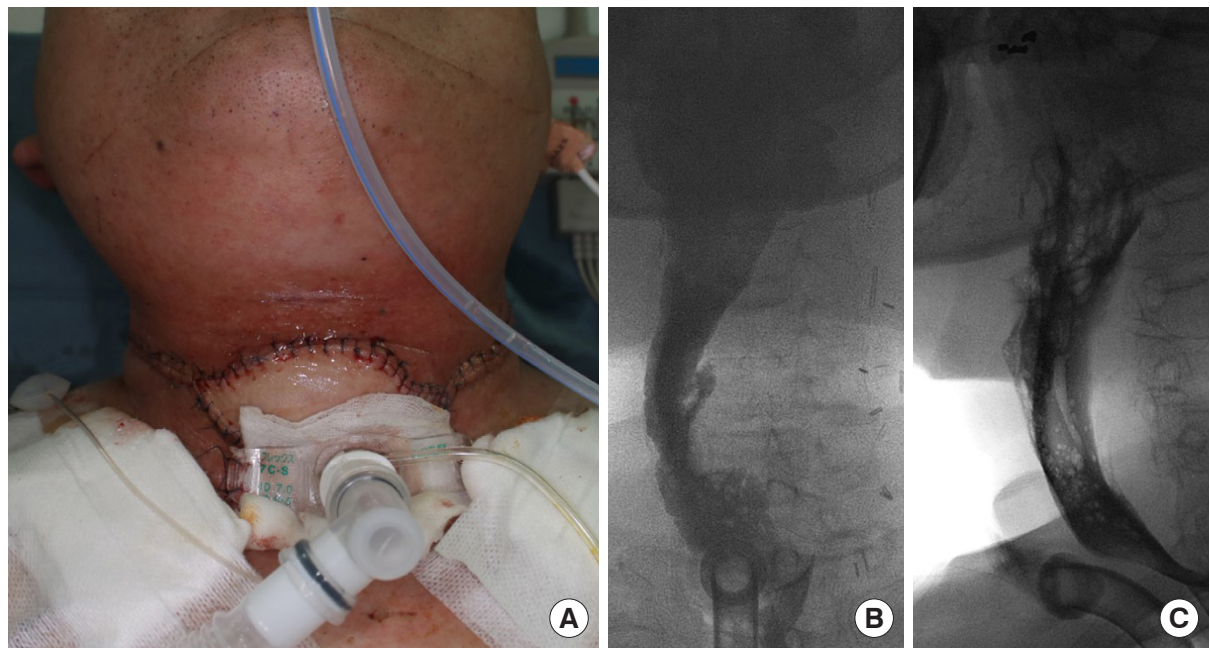

Fig. 4. (A) Successful flap reconstruction of the tracheostomy stroma. (B, C) No leakage or fistula was observed by esophagography at 2 months postoperatively.

vived without problem. At 2 months postoperatively, there was no stricture or fistula on esophagograms (Fig. 4), and the patient could swallow and deglut without problem.

However, at 16 months postoperatively, the patient visited our emergency room with sudden intraoral bleeding. A $1 \mathrm{~cm}$ diameter necrotic region and ulceration were found around the flap margin. Bleeding was controlled by intraarterial embolization, but he died 10 days later due to septic shock following pneumonia.

\section{DISCUSSION}

Flap choice for reconstruction in head and neck cancer is deter- mined by several factors. To obtain good results, appropriate reconstruction methods should be selected according to operator's preference, patient condition, recipient vessel status, receipt of radiotherapy and its dosage, previous surgery, available flap type, and defect site and size [1]. Reconstruction of the esophagus can be done with a local flap and a free flap. Local flaps are usually used with a deltopectoral flap and a pectoralis major myocutaneous flap, and free flaps are usually used with a jejunal flap and a fasciocutaneous flap. Local flaps are limited by size, thickness, and donor site complications and are used in patients with a poor general condition or when it is difficult to harvest free flaps $[5,6]$.

In the case of an extensive and multiple composite defect, 
large defects need reconstruction methods more than two reconstructive areas and 3D functional area. The options for a functional restoration of these types of defects are limited. Therefore they are a challenging problem in head and neck reconstruction. There are many kinds of reconstruction options for complex defect of the head and neck, the combination of a free flap with a locoregional flap, staged reconstruction with multiple free flaps, the combination of free flaps with alloplastic materials and multiple composite and chimeric free flaps can be uses [7-11].

Large surgical excision of the head and neck cancer induced the defect of the greater volume and multiple type tissue such as, skin, mucosa, soft tissue, and bone. Double or multiple free flaps (including such as the fibular free flap, scapular osteocutaneous, iliac crest osteocutaneous free flap) should be first considered in cases of extensive composite defects that cannot be adequately repaired with a single flap $[11,12]$. But a complication rate, flap survival, and donor site morbidity and functional outcome comparing to other reconstruction options are still debated and studied. Comparing to multiple free flaps, in order to reduce morbidity and surgical time and improving a good functional result, one free flap combination with locoregional flaps (such as the pectoralis major, deltopectoral, cervicofacial flap, cervicopectoral flap, posterior scalp flap) are indicated. When resection includes the intraoral lining and functional area such as, tongue, mouth floor, oropharynx and mandible and extended to facial skin and neck, this technique allows the use of a free flap for reconstructing the intraoral lining and mandible, and a locoregional flap for reconstructing the external structures. The facial or neck skin gives optimal aesthetic results because they are similar to the resected tissue in terms of color, texture, hair bearing and thickness [12].

When defects of the esophagus and tracheostomy stroma are present various reconstruction methods are possible. The esophagus can be reconstructed with a jejunal free flap or a fasciocutaneous free flap, and tracheostomy stroma can be reconstructed with a fasciocutaneous free flap or local flap. In the described case, the preoperative plan was devised based on the use of a double free flap, an ALT flap for the esophagus, and a radial forearm flap for tracheostomy stroma. After functional neck dissection, we found only the superior thyroidal artery was available because of previous surgical and radiation vessel injury. Accordingly, we changed the reconstruction method. Because the esophagus and tracheostomy were reasonably close, an ALT flap with a chimeric flap was an option. We achieved successful reconstruction using single chimeric ALT flap for the esophagus and tracheostomy stroma. Depending the surgeon 's preference and presence of the appropriate perfo- rator, other chimeric flap such as, thoracodorsal artery perforator flap (latissimus dorsi), deep inferior epigastric artery perforator flap (rectus abdominis) can be used for this case.

A surgeon should bear in mind that the status of recurrent head and neck cancer may change and be prepared to use any type of flap to address the situation presented.

\section{NOTES}

\section{Conflict of interest}

No potential conflict of interest relevant to this article was reported.

\section{Ethical approval}

The study was approved by the Institutional Review Board of Inha University Hospital (IRB No. 2019-08-021) and performed in accordance with the principles of the Declaration of Helsinki. Written informed consent was obtained.

\section{Patient consent}

The patient provided written informed consent for the publication and the use of his images.

\section{ORCID}

Sae Hwi Ki https://orcid.org/0000-0001-9194-9681

Sung Hwan Ma https://orcid.org/0000-0002-1956-9016

Seung Hyun Sim https://orcid.org/0000-0002-7513-1748

Matthew Seung Suk Choi https://orcid.org/0000-0002-7849-

1861

\section{REFERENCES}

1. Elfeky AE, Nasr WF, Khazbak A, Abdelrahman MS, Allam ZA, Gareer WY, et al. Hypopharyngeal reconstruction: a comparison of three alternatives. Eur Arch Otorhinolaryngol 2015; 272:3045-50.

2. Maldonado AA, Silva AK, Humphries LS, Gottlieb LJ. Complex orofacial reconstruction with the intrinsic chimeric flap. J Reconstr Microsurg 2017;33:233-43.

3. Yazar S, Wei FC, Chen HC, Cheng MH, Huang WC, Lin CH, et al. Selection of recipient vessels in double free-flap reconstruction of composite head and neck defects. Plast Reconstr Surg 2005;115:1553-61.

4. Chen CL, Zenga J, Roland LT, Pipkorn P. Complications of double free flap and free flap combined with locoregional flap in head and neck reconstruction: a systematic review. Head Neck 2018;40:632-46.

5. Nagel TH, Hayden RE. Advantages and limitations of free and 
pedicled flaps in reconstruction of pharyngoesophageal defects. Curr Opin Otolaryngol Head Neck Surg 2014;22:407-13.

6. Richmon JD, Brumund KT. Reconstruction of the hypopharynx: current trends. Curr Opin Otolaryngol Head Neck Surg 2007;15:208-12.

7. Blackwell KE, Buchbinder D, Biller HF, Urken ML. Reconstruction of massive defects in the head and neck: the role of simultaneous distant and regional flaps. Head Neck 1997;19: 620-8.

8. Koshima I. A new classification of free combined or connected tissue transfers: introduction to the concept of bridge, siamese, chimeric, mosaic, and chain-circle flaps. Acta Med Okayama 2001;55:329-32.
9. Boyd JB, Mulholland RS, Davidson J, Gullane PJ, Rotstein LE, Brown $\mathrm{DH}$, et al. The free flap and plate in oromandibular reconstruction: long-term review and indications. Plast Reconstr Surg 1995;95:1018-28.

10. Hallock GG. Further clarification of the nomenclature for compound flaps. Plast Reconstr Surg 2006;117:151e-60e.

11. Hsieh TY, Bewley A. Use of multiple free flaps in head and neck reconstruction. Curr Opin Otolaryngol Head Neck Surg 2019;27:392-400.

12. Bianchi B, Ferri A, Ferrari S, Copelli C, Poli T, Sesenna E. Free and locoregional flap associations in the reconstruction of extensive head and neck defects. Int J Oral Maxillofac Surg 2008; 37:723-9. 